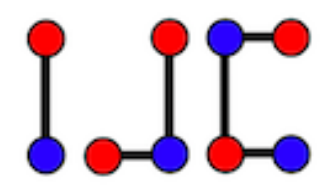

\title{
Exclusive graphs: a new link among labelings
}

\author{
Rikio Ichishima $^{\mathrm{a}}$, Francesc A. Muntaner-Batle ${ }^{\mathrm{b}}$, Akito Oshima ${ }^{\mathrm{b}}$ \\ ${ }^{a}$ Department of Sport and Physical Education, Faculty of Physical Education, Kokushikan University, 7-3-1 \\ Nagayama, Tama-shi, Tokyo, Japan \\ ${ }^{b}$ Graph Theory and Applications Research Group, School of Electrical Engineering and Computer Science, \\ Faculty of Engineering and Built Environment, The University of Newcastle, NSW 2308 Australia \\ ichishim@kokushikan.ac.jp, famb1es@yahoo.es, akitoism@yahoo.co.jp
}

\begin{abstract}
In this paper, we define a strongly felicitous graph to be lower-exclusive, upper-exclusive and exclusive depending on different restrictions for the vertex labels. With these new concepts, we show that the union of finite collection of strongly felicitous graphs, a lower-exclusive one and an upper-exclusive one results in a strongly felicitous graph. We also introduce the concept of decompositional graphs. By means of this, we provide some results involving the cartesian products of exclusive graphs.
\end{abstract}

Keywords: exclusive graph, (strongly) felicitous graph, decompositional graph, cartesian product, union, graph labeling

Mathematics Subject Classification: 05C78, 05C76

DOI: $10.19184 /$ ijc.2019.3.1.1

\section{Introduction}

For any undefined graph theory terminology and notation, the authors refer the reader to the book by Chartrand and Lesniak [1]. All graphs considered in this paper are finite, simple and undirected. The vertex set of a graph $G$ is denoted by $V(G)$, while the edge set of $G$ is denoted by $E(G)$.

For the sake of notational convenience, we will denote the interval of integers $k$ such that $i \leq k \leq j$ by simply writing $[i, j]$.

Received: 19 Feb 2019, Revised: 02 May 2019, Accepted: 12 Jun 2019. 
In 1967, Rosa [13] introduced the notion of $\beta$-valuations. They were later studied by Golomb [8] who called them graceful labelings. This is the term used in the current literature of graph labelings. A graph $G$ of size $q$ is called graceful if there exists an injective function $f: V(G) \rightarrow[0, q]$ such that each $u v \in E(G)$ is labeled $|f(u)-f(v)|$ and the resulting edge labels are distinct. Such a function is called a graceful labeling. Rosa [13] also introduced the notion of $\alpha$-valuations stemming from his interest in graph decompositions. A graceful labeling $f$ is called an $\alpha$-valuation if there exists an integer $\lambda$ (called the boundary value or critical number) so that $\min \{f(u), f(v)\} \leq$ $\lambda<\max \{f(u), f(v)\}$ for each $u v \in E(G)$. Moreover, he pointed out that a graph that admits an $\alpha$-valuation is necessarily bipartite and, consequently, cannot contain a cycle of odd length.

In 1980, Graham and Sloane [7] published the first paper on the concept of harmonious labelings. A graph $G$ of size $q$ is called harmonious if there exists an injective function $f: V(G) \rightarrow \mathbb{Z}_{q}$ such that each $u v \in E(G)$ is labeled $f(u)+f(v)(\bmod q)$ and the resulting edge labels are distinct. Such a function is called a harmonious labeling. If $G$ is a tree, exactly two vertices may be labeled the same. Harmonious graphs naturally arose in the study by them of modular versions of additive bases problems stemming from error-correcting codes.

In 1991, Lee et al. [11] introduced the concept of felicitous labelings. The purpose of this type of labeling is to generalize both harmonious and strongly c-elegant labelings (see [6] for the definition of a $c$-elegant labeling). A graph $G$ of size $q$ is called felicitous if there exists an injective function $f: V(G) \rightarrow \mathbb{Z}_{q+1}$ such that each $u v \in E(G)$ is labeled $f(u)+f(v)(\bmod q)$ and the resulting edge labels are distinct. Such a function is called a felicitous labeling.

In 2003, Figueroa-Centeno et al. [4] defined a felicitous graph $G$ with a felicitous labeling $f$ to be strongly felicitous if there exists an integer $\lambda$ (called the characteristic of $f$ ) so that for each $u v \in E(G)$, $\min \{f(u), f(v)\} \leq \lambda<\max \{f(u), f(v)\}$. In such a case, $f$ is called a strongly felicitous labeling. It is clearly true that if a graph $G$ has a strongly felicitous labeling $f$ with characteristic $\lambda$, then $G$ is bipartite with partite sets $\{v \in V(G): f(v) \leq \lambda\}$ and $\{v \in V(G): f(v)>\lambda\}$. It has been established in [4] that for every graph $G$ with $|E(G)| \geq$ $|V(G)|-1, G$ is strongly felicitous if and only if $G$ has an $\alpha$-valuation. However, if $G$ is strongly felicitous and has an $\alpha$-valuation, then $G$ always meets the condition $|E(G)| \geq|V(G)|-1$; hence, we obtain the following extension of the mentioned result.

Lemma 1.1. For every graph $G, G$ is strongly felicitous if and only if $G$ has an $\alpha$-valuation.

We close this section with suggestions for further reading. The previously mentioned paper by Rosa [13] would have major influence on the area of graph labelings. Since then numerous types of graph labelings have been introduced and studied. These have been described in a dynamic survey written by Gallian [6]. Readers interested in more information on felicitous graphs may consult the results in $[3,5,9,12,14,15]$.

\section{Results on Unions of Graphs}

To present the results in this section, it is useful to introduce some additional notation. For a vertex labeling $f$ of a graph $G$ and a nonempty subset $S$ of $V(G)$, we will denote the set of vertex labels $\{f(v): v \in S\}$ by writing $f(S)$ and we will write $f(G)$ instead of $f(V(G))$. We will also 
utilize the following notation: if $A \subseteq \mathbb{Z}$ and $b \in \mathbb{Z}$, then $A+b=\{a+b: a \in A\}$, where $\mathbb{Z}$ is the set of all integers. It is now possible to present the next result.

Lemma 2.1. If $G$ is a graph that has a strongly felicitous labeling $f$ with characteristic $\lambda$, then 0 , $\lambda, \lambda+1$ and $|E(G)|$ are elements of the set $f(G)$, and

$$
\{f(x)+f(y): x y \in E(G)\}=[1,|E(G)|]+\lambda .
$$

Proof. If a graph $G$ has a strongly felicitous labeling $f$ with characteristic $\lambda$, then $G$ has the partite sets $X$ and $Y$ such that

$$
X=\{v \in V(G): f(v) \leq \lambda\} \text { and } Y=\{v \in V(G): f(v)>\lambda\} .
$$

In the proof of Lemma 1.1, the vertex labeling $g: V(G) \rightarrow[0,|E(G)|]$ such that $g(x)=f(x)$ for all $x \in X$ and $g(y)=\lambda+|E(G)|+1-f(y)$ for all $y \in Y$ has been shown to be an $\alpha$-valuation (see [4]). By the definition of an $\alpha$-valuation, it is true that $0, \lambda, \lambda+1$ and $|E(G)|$ are the elements of the set $g(G)$. This implies that $0, \lambda, \lambda+1$ and $|E(G)|$ are also the elements of the set $f(G)$. By the definition of $g$, it is clear that

$$
\begin{aligned}
f(x)+f(y) & =g(x)+(\lambda+|E(G)|+1-g(y)) \\
& =\lambda+|E(G)|+1-g(y)+g(x) \\
& =\lambda+|E(G)|+1-|g(y)-g(x)|
\end{aligned}
$$

for each $x y \in E(G)$, where $x \in X$ and $y \in Y$. Moreover, since $g$ is an $\alpha$-valuation of $G$, it follows that

$$
\{|g(y)-g(x)|: x y \in E(G)\}=[1,|E(G)|]
$$

which implies that

$$
\{f(x)+f(y): x y \in E(G)\}=[1,|E(G)|]+\lambda .
$$

Lemma 2.1 gives rise to the following concepts. A strongly felicitous labeling $f$ of a graph $G$ with characteristic $\lambda$ is called lower-exclusive if $\lambda>1$ and $1 \notin f(G)$, and upper-exclusive if $\lambda>2$ and $\lambda-1 \notin f(G)$. If $f$ is both lower-exclusive and upper-exclusive with $\lambda>2$, then $f$ is called exclusive. A graph with a strongly felicitous labeling that is lower-exclusive is called lower-exclusive, while a graph with a strongly felicitous labeling that is upper-exclusive is called upper-exculsive. Thus, a graph with a strongly felicitous labeling that is exclusive is called exclusive.

For two graphs $G_{1}$ and $G_{2}$ with disjoint vertex sets, the union $G \cong G_{1} \cup G_{2}$ can be defined with $V(G)=V\left(G_{1}\right) \cup V\left(G_{2}\right)$ and $E(G)=E\left(G_{1}\right) \cup E\left(G_{2}\right)$. If a graph $G$ consists of $m$ disjoint copies of a graph $H$, then we simply write $G \cong m H$, where $m \geq 2$. For the rest of this section, we concern with some results involving the union of strongly felicitous graphs. We start with the following result.

Theorem 2.1. If $G_{1}$ is upper-exclusive and $G_{2}$ is lower-exclusive, then $G \cong G_{1} \cup G_{2}$ is strongly felicitous. 
Proof. Consider the strongly felicitous labelings $f_{1}$ and $f_{2}$ of $G_{1}$ and $G_{2}$ with characteristic $\lambda_{1}$ and $\lambda_{2}$ that are upper-exclusive and lower-exclusive, respectively. Then $G_{1}$ is bipartite with partite sets $X_{1}$ and $Y_{1}$ such that

$$
X_{1}=\left\{v \in V\left(G_{1}\right): f_{1}(v) \leq \lambda_{1}\right\} \text { and } Y_{1}=\left\{v \in V\left(G_{1}\right): f_{1}(v)>\lambda_{1}\right\},
$$

whereas $G_{2}$ is bipartite with partite sets $X_{2}$ and $Y_{2}$ such that

$$
X_{2}=\left\{v \in V\left(G_{2}\right): f_{2}(v) \leq \lambda_{2}\right\} \text { and } Y_{2}=\left\{v \in V\left(G_{2}\right): f_{2}(v)>\lambda_{2}\right\} .
$$

Now, consider the vertex labeling $g: V(G) \rightarrow\left[0,\left|E\left(G_{1}\right)\right|+\left|E\left(G_{2}\right)\right|\right]$ such that

$$
g(v)= \begin{cases}f_{1}(v) & \text { if } v \in X_{1}, \\ f_{1}(v)+\lambda_{2}-1 & \text { if } v \in Y_{1}, \\ f_{2}(v)+\lambda_{1}-1 & \text { if } v \in X_{2} \\ f_{2}(v)+\left|E\left(G_{1}\right)\right| & \text { if } v \in Y_{2}\end{cases}
$$

Then $g$ extends to a strongly felicitous labeling of $G$. To see this, we first compute the edge labels induced by $g(u)+g(v)$ for each $u v \in E(G)$. Observe that

$$
g(x)+g(y)=f_{1}(x)+f_{1}(y)+\lambda_{2}-1
$$

for all $x y \in E\left(G_{1}\right)$, where $x \in X_{1}$ and $y \in Y_{1}$. Also, observe that

$$
g(x)+g(y)=f_{2}(x)+f_{2}(y)+\lambda_{1}+\left|E\left(G_{1}\right)\right|-1
$$

for all $x y \in E\left(G_{2}\right)$, where $x \in X_{2}$ and $y \in Y_{2}$. This together with Lemma 2.1 implies that

$$
\left\{f_{1}(x)+f_{1}(y): x y \in E\left(G_{1}\right)\right\}=\left[1,\left|E\left(G_{1}\right)\right|\right]+\lambda_{1}
$$

and

$$
\left\{f_{2}(x)+f_{2}(y): x y \in E\left(G_{2}\right)\right\}=\left[1,\left|E\left(G_{2}\right)\right|\right]+\lambda_{2},
$$

respectively. This in turn implies that

$$
\left\{g(x)+g(y): x y \in E\left(G_{1}\right)\right\}=\left[1,\left|E\left(G_{1}\right)\right|\right]+\lambda_{1}+\lambda_{2}-1
$$

and

$$
\left\{g(x)+g(y): x y \in E\left(G_{2}\right)\right\}=\left[\left|E\left(G_{1}\right)\right|+1,\left|E\left(G_{1}\right)\right|+\left|E\left(G_{2}\right)\right|\right]+\lambda_{1}+\lambda_{2}-1 .
$$

Thus, it follows that

$$
\{g(x)+g(y): x y \in E(G)\}=[1,|E(G)|]+\lambda_{1}+\lambda_{2}-1,
$$

since $|E(G)|=\left|E\left(G_{1}\right)\right|+\left|E\left(G_{2}\right)\right|$. In order to show that $g$ is indeed an injective function, it suffices to verify that $g\left(X_{1}\right) \cap g\left(X_{2}\right)=\emptyset$, since

$$
0 \leq g(v) \leq\left|E\left(G_{1}\right)\right|+\left|E\left(G_{2}\right)\right|=|E(G)|
$$


for all $v \in V(G)$, and

$$
\begin{array}{ll}
g\left(X_{1}\right) \subseteq\left[0, \lambda_{1}\right], & g\left(X_{2}\right) \subseteq\left[\lambda_{1}-1, \lambda_{1}+\lambda_{2}-1\right], \\
g\left(Y_{1}\right) \subseteq\left[\lambda_{1}+\lambda_{2}, \lambda_{2}+\left|E\left(G_{1}\right)\right|-1\right], & g\left(Y_{2}\right) \subseteq\left[\lambda_{2}+\left|E\left(G_{1}\right)\right|,|E(G)|\right] .
\end{array}
$$

First, observe that the largest element of $g\left(X_{1}\right)$ is $\lambda_{1}$ and the next smallest element is less than $\lambda_{1}-1$, since $f_{1}$ is upper-exclusive. Also, observe that the smallest element of $g\left(X_{2}\right)$ is $\lambda_{1}-1$, and the next largest element is greater than $\lambda_{1}$, since $f_{2}$ is lower-exclusive. Thus, $g\left(X_{1}\right) \cap g\left(X_{2}\right)=\emptyset$. It remains to observe that $G$ is bipartite with the partite sets $X_{1} \cup X_{2}$ and $Y_{1} \cup Y_{2}$, and the largest element of $g\left(X_{1} \cup X_{2}\right)$ is $\lambda_{1}+\lambda_{2}-1$. Therefore, we conclude that $g$ is a strongly felicitous labeling of $G$ with characteristics $\lambda_{1}+\lambda_{2}-1$.

In the proof of Theorem 2.1, notice that if $f_{1}$ is exclusive, then $\lambda_{1}>2$ and $1 \notin f_{1}\left(G_{1}\right)$, which implies that $\lambda_{1}+\lambda_{2}-1>1$ and $1 \notin g(G)$. Thus, we have the following result.

Corollary 2.1. If $G_{1}$ is exclusive and $G_{2}$ is lower-exclusive, then $G \cong G_{1} \cup G_{2}$ is lower-exclusive.

In the proof of Theorem 2.1, notice that if $f_{2}$ is exclusive, then $\lambda_{2}>2$ and $\lambda_{2}-1 \notin f_{2}\left(G_{2}\right)$. This implies that $\lambda_{1}+\lambda_{2}-1>1$. However, $\left(\lambda_{1}-1\right)+\lambda_{2}-1=\lambda_{1}+\lambda_{2}-2 \notin g\left(X_{2}\right)$. Thus, we have the following result.

Corollary 2.2. If $G_{1}$ is upper-exclusive and $G_{2}$ is exclusive, then $G \cong G_{1} \cup G_{2}$ is upper-exclusive.

In the proof of Theorem 2.1, notice that if either $f_{1}$ or $f_{2}$ is exclusive, then $\lambda_{1}+\lambda_{2}-1>2$. This together with Corollaries 2.1 and 2.2 gives us the following result.

Corollary 2.3. If $G_{1}$ and $G_{2}$ are exclusive, then $G \cong G_{1} \cup G_{2}$ is exclusive.

As an immediate consequence of Corollary 2.3, we have the following result.

Corollary 2.4. Let $n$ be an integer with $n \geq 3$. If $G_{1}, G_{2}, \ldots, G_{n}$ are exclusive, then $\bigcup_{i=1}^{n} G_{i}$ is exclusive.

Combining Theorem 2.1 with Corollaries 2.2 and 2.3, we arrive at the following result.

Corollary 2.5. Let $n$ be an integer with $n \geq 3$. If $G_{1}$ is upper-exclusive, $G_{2}, G_{3}, \ldots, G_{n-1}$ are exclusive and $G_{n}$ is lower-exclusive, then $\bigcup_{i=1}^{n} G_{i}$ is strongly felicitous.

Proof. Consider such graphs $G_{1}, G_{2}, \ldots, G_{n}$. It follows from Corollary 2.3 that $G_{2} \cup G_{3} \cup \cdots \cup$ $G_{n-1}$ is exclusive. By Corollary 2.2, this together with our assumption implies that $G_{1} \cup G_{2} \cup$ $\cdots \cup G_{n-1}$ is upper-exclusive. It is now immediate from Theorem 2.1 that $G_{1} \cup G_{2} \cup \cdots \cup G_{n}$ is strongly felicitous.

The proof technique involved in the proof of Theorem 2.1 can be generalized as described in the following result. 
Theorem 2.2. Let $G_{i}$ be a graph that has a strongly felicitous labeling $f_{i}$ with characteristic $\lambda_{i}$, and let $X_{i}=\left\{v \in V\left(G_{i}\right): f_{i}(v) \leq \lambda_{i}\right\}$ for $i=1,2$. If there exists an integer $\mu$ with $0<\mu<\lambda_{1}$ such that

$$
\left\{f_{1}(v): v \in X_{1}\right\} \cap\left\{f_{2}(v)+\lambda_{1}-\mu: v \in X_{2}\right\}=\emptyset,
$$

then $G \cong G_{1} \cup G_{2}$ is strongly felicitous.

Proof. Let $G_{i}$ have partite sets $X_{i}$ and $Y_{i}$, where

$$
X_{i}=\left\{v \in V\left(G_{i}\right): f_{i}(v) \leq \lambda_{i}\right\} \text { and } Y_{i}=\left\{v \in V\left(G_{i}\right): f_{i}(v)>\lambda_{i}\right\} \text { for } i=1,2 .
$$

Further, let $\lambda_{i}^{\prime}=\lambda_{i}-\mu$ for $i=1,2$, and consider the vertex labeling $g: V(G) \rightarrow\left[0,\left|E\left(G_{1}\right)\right|+\left|E\left(G_{2}\right)\right|\right]$ such that

$$
g(v)= \begin{cases}f_{1}(v) & \text { if } v \in X_{1}, \\ f_{1}(v)+\lambda_{2}^{\prime} & \text { if } v \in Y_{1}, \\ f_{2}(v)+\lambda_{1}^{\prime} & \text { if } v \in X_{2}, \\ f_{2}(v)+\left|E\left(G_{1}\right)\right| & \text { if } v \in Y_{2} .\end{cases}
$$

To show that $g$ is indeed a strongly felicitous labeling of $G$, we first compute the edge labels induced by $g(u)+g(v)$ for each $u v \in E(G)$. Notice that

$$
g(x)+g(y)=f_{1}(x)+f_{1}(y)+\lambda_{2}^{\prime}
$$

for all $x y \in E\left(G_{1}\right)$ and

$$
g(x)+g(y)=f_{2}(x)+f_{2}(y)+\left|E\left(G_{1}\right)\right|+\lambda_{1}^{\prime}
$$

for all $x y \in E\left(G_{2}\right)$. This together with Lemma 2.1 implies that

$$
\left\{f_{1}(x)+f_{1}(y): x y \in E\left(G_{1}\right)\right\}=\left[1,\left|E\left(G_{1}\right)\right|\right]+\lambda_{1}
$$

and

$$
\left\{f_{2}(x)+f_{2}(y): x y \in E\left(G_{2}\right)\right\}=\left[1,\left|E\left(G_{2}\right)\right|\right]+\lambda_{2} .
$$

This in turn implies that

$$
\left\{g(x)+g(y): x y \in E\left(G_{1}\right)\right\}=\left[1,\left|E\left(G_{1}\right)\right|\right]+\lambda_{1}+\lambda_{2}^{\prime}
$$

and

$$
\left\{g(x)+g(y): x y \in E\left(G_{2}\right)\right\}=\left[\left|E\left(G_{1}\right)\right|+1,|E(G)|\right]+\lambda_{1}^{\prime}+\lambda_{2},
$$

since $|E(G)|=\left|E\left(G_{1}\right)\right|+\left|E\left(G_{2}\right)\right|$. However, $\lambda_{1}^{\prime}+\lambda_{2}=\lambda_{1}+\lambda_{2}^{\prime}$ and so

$$
\left\{g(x)+g(y): x y \in E\left(G_{2}\right)\right\}=\left[\left|E\left(G_{1}\right)\right|+1,|E(G)|\right]+\lambda_{1}+\lambda_{2}^{\prime} .
$$

By assumption of the theorem,

$$
\left\{f_{1}(v): v \in X_{1}\right\} \cap\left\{f_{2}(v)+\lambda_{1}^{\prime}: v \in X_{2}\right\}=\emptyset,
$$


which implies that $g\left(X_{1}\right) \cap g\left(X_{2}\right)=\emptyset$. This together with the facts that $\lambda_{1}^{\prime}+\lambda_{2}=\lambda_{1}+\lambda_{2}^{\prime}$,

$$
\lambda_{2}^{\prime}+\left|E\left(G_{1}\right)\right|=\lambda_{2}-\mu+\left|E\left(G_{1}\right)\right|<\lambda_{2}+\left|E\left(G_{1}\right)\right|
$$

and

$$
\begin{array}{ll}
g\left(X_{1}\right) \subseteq\left[0, \lambda_{1}\right], & g\left(X_{2}\right) \subseteq\left[\lambda_{1}^{\prime}, \lambda_{1}^{\prime}+\lambda_{2}\right] \\
g\left(Y_{1}\right) \subseteq\left[\lambda_{1}+\lambda_{2}^{\prime}+1, \lambda_{2}^{\prime}+\left|E\left(G_{1}\right)\right|\right], & g\left(Y_{2}\right) \subseteq\left[\lambda_{2}+\left|E\left(G_{1}\right)\right|+1,|E(G)|\right]
\end{array}
$$

implies that $g$ is an injective function. It remains to observe that $G$ is bipartite with partite sets $X_{1} \cup X_{2}$ and $Y_{1} \cup Y_{2}$, the largest element of $g\left(X_{1} \cup X_{2}\right)$ is $\lambda_{1}^{\prime}+\lambda_{2}$ and

$$
\lambda_{1}^{\prime}+\lambda_{2}=\left(\lambda_{1}-\mu\right)+\lambda_{2}>\lambda_{2}>1
$$

by assumption of the theorem. Therefore, $g$ is a strongly felicitous labeling of $G$ with characteristic $\lambda_{1}^{\prime}+\lambda_{2}$, which completes the proof.

\section{Results on Cartesian Products}

The cartesian product $G \cong G_{1} \times G_{2}$ of two graphs $G_{1}$ and $G_{2}$ with disjoint vertex sets is the graph with vertex set $G \cong V\left(G_{1}\right) \times V\left(G_{2}\right)$ such that two vertices $\left(u_{1}, u_{2}\right)$ and $\left(v_{1}, v_{2}\right)$ of $G$ are adjacent if and only if either

$$
u_{1}=v_{1} \text { and } u_{2} v_{2} \in E\left(G_{2}\right)
$$

or

$$
u_{2}=v_{2} \text { and } u_{1} v_{1} \in E\left(G_{1}\right) .
$$

A class of graphs that is involved in this section can be described in terms of cartesian product. The hypercube $Q_{n}$ can be defined recursively as $Q_{1} \cong K_{2}$ and $Q_{n} \cong Q_{n-1} \times K_{2}$ for any integer $n \geq 2$.

In the following, we show how to recursively create exclusive graphs from certain kinds of existing strongly felicitous graphs. To present this, it is convenient to introduce another concept. A strongly felicitous labeling $f$ of a bipartite graph $G$ with partite sets $X$ and $Y$ such that $|X|=$ $|Y|=s$ and $|E(G)|=2 t+s$ for some positive integers $s$ and $t$ is called a decompositional labeling if $f$ satisfies the following conditions:

1. There exists a positive integer $\lambda$ such that $f(x) \leq \lambda$ for all $x \in X$ and $f(y)>\lambda$ for all $y \in Y$, where $t-s \leq \lambda \leq t+s-1$.

2. There exists a positive integer $\lambda$ such that the edge labels induced by $f(u)+f(v)$ for each $u v \in E(G)$ are decomposed into three sets $[\lambda+1, \lambda+t],[\lambda+t+1, \lambda+t+s]$ and $[\lambda+t+s+1, \lambda+2 t+s]$.

3. There exists an involution $\pi$, which is an automorphism of $G$ such that $\pi$ exchanges $X$ and $Y$.

4. The $s$ edges $x \pi(x) \in E(G)$ for all $x \in X$ have as their labels the elements of the set $[\lambda+t+1, \lambda+t+s]$. 
A graph that has a decompositional labeling is called decompositional. We are now in a position to present the following result.

Theorem 3.1. If $G$ is a decompositional graph that is exclusive, then so is $G \times K_{2}$.

Proof. We utilize the notation given in the definition of a decompositional labeling, and assume that $G$ has a decompositional labeling $f$ with characteristic $\lambda$ that is exclusive. Then $G$ is bipartite with partite sets $X$ and $Y$ of the same cardinality $s$ such that $f(x) \leq \lambda$ for all $x \in X$ and $f(y)>\lambda$ for all $y \in Y$, where $t-s \leq \lambda \leq t+s-1$, and the number of edges is $2 t+s$. Also, there exists an involution $\pi$, which is an automorphism of $G$ such that $\pi$ exchanges $X$ and $Y$. Moreover, the $s$ edges $x \pi(x) \in E(G)$ for all $x \in X$ have as labels the integers of $[\lambda+t+1, \lambda+t+s]$. If we let $X=\left\{v_{i}: i \in[1, s]\right\}$ and $Y=\left\{v_{i}: i \in[s+1,2 s]\right\}$ so that $\pi\left(v_{i}\right)=v_{2 s+1-i}$ for each $i \in[1, s]$, then $\pi$ is an involutive automorphism of $G$, and we have

$$
\left\{f\left(v_{i}\right)+f\left(\pi\left(v_{i}\right)\right): i \in[1, s]\right\}=[\lambda+t+1, \lambda+t+s] .
$$

Since $f$ is exclusive and has the characteristic $\lambda$, it follows that $s \in[2, \lambda]$. Let $H \cong G \times K_{2}$, and construct the graph $H$ by using the decomposition $H \cong H_{1} \oplus H_{2} \oplus H_{3}$ with

$$
\begin{aligned}
& V\left(H_{1}\right)=\left\{w_{i}: i \in[1, s]\right\} \cup\left\{w_{i}: i \in[2 s+1,3 s]\right\}, \\
& V\left(H_{2}\right)=\left\{w_{i}: i \in[1,4 s]\right\} \\
& V\left(H_{3}\right)=\left\{w_{i}: i \in[s+1,2 s]\right\} \cup\left\{w_{i}: i \in[3 s+1,4 s]\right\}, \\
& E\left(H_{1}\right)=\left\{w_{i} w_{2 s+j}: v_{i} v_{s+j} \in E(G), i \in[1, s] \text { and } j \in[1, s]\right\}, \\
& E\left(H_{2}\right)=\left\{w_{i} w_{4 s+1-i}: i \in[1,2 s]\right\}, \\
& E\left(H_{3}\right)=\left\{w_{s+i} w_{3 s+j}: v_{i} v_{s+j} \in E(G), i \in[1, s] \text { and } j \in[1, s]\right\} .
\end{aligned}
$$

In this construction, notice that $H_{1}$ and $H_{3}$ are isomorphic to $G, H_{2}$ is isomorphic to $2 s K_{2}$, and $H$ is represented as a bipartite graph with partite sets

$$
X^{\prime}=\left\{w_{i}: i \in[1,2 s]\right\} \text { and } Y^{\prime}=\left\{w_{i}: i \in[2 s+1,4 s]\right\}
$$

of the same cardinality $2 s$, and the number of edges is $4 t+4 s$. Note that if we define a permutation $\pi^{\prime}$ of $V(H)$ by

$$
\pi^{\prime}\left(w_{i}\right)=w_{4 s+1-i}(i \in 1,4 s),
$$

then $\pi^{\prime}$ is an involutive automorphism of $H$, and we have

$$
E\left(H_{2}\right)=\left\{w_{i} \pi^{\prime}\left(w_{i}\right): i \in[1,2 s]\right\} .
$$

With the preceding construction in hand, we now consider the vertex labeling $g: V(H) \rightarrow$ $[0,4 t+4 s]$ such that

$$
\begin{aligned}
g\left(w_{i}\right) & =f\left(v_{i}\right), \\
g\left(w_{s+i}\right) & =f\left(v_{i}\right)+t+s, \\
g\left(w_{2 s+i}\right) & =f\left(v_{s+i}\right)+t+s, \\
g\left(w_{3 s+i}\right) & =f\left(v_{s+i}\right)+2 t+3 s
\end{aligned}
$$


for each $i \in[1, s]$. Then $g$ extends to a decompositional labeling of $G$ with characteristic $\lambda+t+s$. To see this, notice that

$$
\left\{g(x): x \in X^{\prime}\right\} \subseteq[0, \lambda+t+s]
$$

and

$$
\left\{g(y): y \in Y^{\prime}\right\} \subseteq[\lambda+t+s+1,4 t+4 s]
$$

since

$$
\{f(x): x \in X\} \subseteq[0, \lambda] \text { and }\{f(y): y \in Y\} \subseteq[\lambda+1,2 t+s] .
$$

This implies that $g$ is an injective function. Next, notice that for the edges in $H_{1}$ and $H_{3}$, we have

$$
g\left(w_{i}\right)+g\left(w_{2 s+j}\right)=f\left(v_{i}\right)+f\left(v_{s+j}\right)+t+s
$$

and

$$
g\left(w_{s+i}\right)+g\left(w_{3 s+j}\right)=f\left(v_{i}\right)+f\left(v_{s+j}\right)+3 t+4 s,
$$

respectively. The last two equations together with Lemma 2.1 imply that

$$
\left\{g(x)+g(y): x y \in E\left(H_{1}\right)\right\}=[\lambda+t+s+1, \lambda+3 t+2 s]
$$

and

$$
\left\{g(x)+g(y): x y \in E\left(H_{3}\right)\right\}=[\lambda+3 t+4 s+1, \lambda+5 t+5 s] .
$$

Finally, notice that the edges in $H_{2}$ are of two types, namely, either of the form $w_{i} w_{4 s+1-i}$ or $w_{s+i} w_{3 s+1-i}$, where $i \in[1, s]$. Thus, we have

$$
g\left(w_{i}\right)+g\left(w_{4 s+1-i}\right)=f\left(v_{i}\right)+f\left(v_{2 s+1-i}\right)+2 t+3 s
$$

and

$$
g\left(w_{s+i}\right)+g\left(w_{3 s+1-i}\right)=f\left(v_{i}\right)+f\left(v_{2 s+i-1}\right)+2 t+2 s .
$$

The last two equations and the fact that

$$
\left\{f\left(v_{i}\right)+f\left(v_{2 s+1-i}\right): i \in[1, s]\right\}=[\lambda+t+1, \lambda+t+s]
$$

imply that

$$
\left\{g\left(w_{i}\right)+g\left(w_{4 s+1-i}\right): i \in[1, s]\right\}=[\lambda+3 t+3 s+1, \lambda+3 t+4 s]
$$

and

$$
\left\{g\left(w_{s+i}\right)+g\left(w_{3 s+1-i}\right): i \in[1, s]\right\}=[\lambda+3 t+2 s+1, \lambda+3 t+3 s],
$$

which implies that

$$
\left\{g(x)+g(y): x y \in E\left(H_{2}\right)\right\}=[\lambda+3 t+2 s+1, \lambda+3 t+4 s] .
$$

Therefore, $g$ is a decompositional labeling of $H$ with characteristic $\lambda+t+s$.

It remains to show that $g$ is indeed exclusive. First, assume, to the contrary, that there exists a vertex $v \in V(H)$ such that $g(v)=1$. Then $f(v)=1$, which contradicts the fact that $f$ is exclusive. Next, assume, to the contrary, that there exists a vertex $v \in V(H)$ such that $g(v)=$ $\lambda+t+s-1$. Then $f(v)=\lambda-1$, which again contradicts the fact that $f$ is exclusive. It is now immediate that $\lambda+t+s>2$, since $f$ is exclusive. Therefore, $H$ is exclusive, which completes the proof. 
If we apply Theorem 3.1 repeatedly, then we obtain the following result as announced in the beginning of this section.

Theorem 3.2. Let $n$ be a positive integer. If $G$ is a decompositional graph that is exclusive, then so is $G \times Q_{n}$.

It is important to notice that the preceding two theorems affirmatively answer the following problems posed by Figueroa-Centeno and Ichishima [2].

Problem 1. Let $\mathcal{F}$ be a family of felicitous graphs. For which felicitous graphs $G, G \times K_{2} \in \mathcal{F}$ ?

Problem 2. Let $\mathcal{F}$ be a family of felicitous graphs. For which felicitous graphs $G$ and positive integers $n, G \times Q_{n} \in \mathcal{F}$ ?

\section{Applications}

In this section, we provide some results obtained by applying Theorem 3.2. We begin by considering the exclusive property of complete bipartite graphs. The labeling presented in the following theorem is essentially the one given by Lee et al. [11].

Theorem 4.1. For every two integers $m$ and $n$ with $2 \leq n<m$, the complete bipartite graph $K_{m, n}$ is exclusive.

Proof. Let $K_{m, n}$ have partite sets $X$ and $Y$, where $|X|=m$ and $|Y|=n$. Label the vertices of $X$ with $0, n, \ldots,(m-1) n$ and the vertices of $Y$ with $(m-1) n+1,(m-1) n+2, \ldots, m n$. Certainly, this produces a strongly felicitous labeling of $K_{m, n}$ with characteristic $(m-1) n$ that is exclusive.

As a consequence of Theorem 3.2, we have the following result.

Theorem 4.2. For every two integers $m$ and $n$ with $m \geq 2$ and $n \geq 1$, the graph $K_{m, 2} \times Q_{n}$ is exclusive.

Proof. In light of Theorem 3.2, it suffices to show that the graph $K_{m, 2} \times K_{2}$ is decompositional with the exclusive property for every integer $m \geq 2$. Construct the graph $G \cong K_{m, 2} \times K_{2}$ by using the decomposition $G \cong H_{1} \oplus H_{2} \oplus H_{3}$ with

$$
\begin{aligned}
& V\left(H_{1}\right)=\left\{x_{i}: i \in[1, m+2]\right\}, \\
& V\left(H_{2}\right)=\left\{x_{i}: i \in[1, m+2]\right\} \cup\left\{y_{i}: i \in[1, m+2]\right\}, \\
& V\left(H_{3}\right)=\left\{y_{i}: i \in[1, m+2]\right\}, \\
& E\left(H_{1}\right)=\left\{x_{1} x_{i}: i \in[2, m+1]\right\} \cup\left\{x_{i} x_{m+2}: i \in[2, m+1]\right\}, \\
& E\left(H_{2}\right)=\left\{x_{i} y_{i}: i \in[1, m+2]\right\}, \\
& E\left(H_{3}\right)=\left\{y_{1} y_{i}: i \in[2, m+1]\right\} \cup\left\{y_{i} y_{m+2}: i \in[2, m+1]\right\} .
\end{aligned}
$$

Observe that $G$ is represented as a bipartite graph of size $5 m+2$ with partite sets

$$
U=\left\{x_{1}, x_{m+2}\right\} \cup\left\{y_{i}: i \in[2, m+1]\right\} \text { and } V=\left\{x_{i}: i \in[2, m+1]\right\} \cup\left\{y_{1}, y_{m+2}\right\}
$$


of the same cardinality $m+2$. Note that if we define a permutation $\pi$ of $V(G)$ by

$$
\pi\left(x_{i}\right)=y_{i}(i \in[1, m+2]),
$$

then $\pi$ is an involutive automorphism of $G$, and we have

$$
E\left(H_{2}\right)=\left\{x_{i} \pi\left(x_{i}\right): i \in[1, m+2]\right\} .
$$

With the preceding construction in hand, we now consider the vertex labeling $f: V(G) \rightarrow$ $[0,5 m+2]$ such that

$$
f(w)= \begin{cases}m & \text { if } w=x_{1} \\ 3 m-1+i & \text { if } w=x_{i} \text { and } i \in[2, m+1] \\ 0 & \text { if } w=x_{m+2} \\ 5 m+2 & \text { if } w=y_{1}, \\ 3 m+4-2 i & \text { if } w=y_{i} \text { and } i \in[2, m+1] \\ 5 m+1 & \text { if } w=y_{m+2}\end{cases}
$$

Then $f$ extends to a decompositional labeling of $G$ with characteristic $3 m$ that is exclusive. To see that $f$ is an injective function and $1,3 m-1 \notin f(G)$, observe that

$$
f(U)=\{0, m\} \cup[m+2,3 m] \backslash\{3 m+1-2 i: i \in[1, m]\}
$$

and

$$
f(V)=[3 m+1,4 m] \cup\{5 m+1,5 m+2\} .
$$

Also, observe that

$$
\begin{aligned}
& \left\{f(u)+f(v): u v \in E\left(H_{1}\right)\right\} \\
& \left\{f\left(x_{i}\right)+f\left(y_{i}\right): i \in[1, m+2]\right\} \quad=[3 m+1,5 m] \\
& \left\{f(u)+f(v): u v \in E\left(H_{2}\right)\right\} \quad=[6 m+1,6 m+2],
\end{aligned}
$$

It remains to observe that $3 m>2$ for every positive integer $m$. Therefore, $f$ is a decompositional labeling of $G$ with characteristic $3 m$ that is exclusive, and the desired result follows from Theorem 3.2 .

It is known from [2] that the hypercube $Q_{n}$ is felicitous for every integer $n \geq 2$. It is also straightforward to see that $Q_{2} \cong K_{2,2}$ is not exclusive; however, by letting $m=2$ in Theorem 4.2, we obtain a characterization of exclusive hypercubes.

Corollary 4.1. The hypercube $Q_{n}$ is exclusive if and only if $n \geq 3$.

From Corollaries 2.3 and 4.1, we have the following two results.

Corollary 4.2. For every two integers $m$ and $n$ with $m \geq n \geq 3$, the graph $Q_{m} \cup Q_{n}$ is exclusive.

Corollary 4.3. For every two integers $m$ and $n$ with $m \geq 1$ and $n \geq 3$, the graph $m Q_{n}$ is exclusive. 
It was shown by Kotzig [10] that every hypercube has an $\alpha$-valuation. Now, in light of Lemma 2.2, Corollaries 4.2 and 4.3 give rise to the following two results.

Corollary 4.4. For every two integers $m$ and $n$ with $m \geq n \geq 3$, the graph $Q_{m} \cup Q_{n}$ has an $\alpha$-valuation.

Corollary 4.5. For every two integers $m$ and $n$ with $m \geq 1$ and $n \geq 3$, the graph $m Q_{n}$ has an $\alpha$-valuation.

\section{Conclusions}

In this paper, we have defined what it means for a strongly felicitous labeling to be exclusive, along with the weaker properties of being lower-exclusive and upper-exclusive. After that, we have proven that the union of two graphs are strongly felicitous if one of these is upper-exclusive and another one is lower-exclusive. This can be extended to obtain the result that the union of a finite collection of strongly felicitous graphs, all exclusive except one that may be upper-exclusive and another one lower-exclusive, admits a strongly felicitous labeling.

We have also introduced the notion of decompositional labelings and have provided a recursive construction to create exclusive graphs. As consequences of this result, various graphs have been shown to be exclusive including most hypercubes. From Lemma 2.1, we know that if $G$ is a strongly felicitous graph, then $G$ has an $\alpha$-valuation. Therefore, we conclude that the work presented in this paper has considerably enlarged the classes of graphs known to be strongly felicitous as well as graphs with $\alpha$-valuations.

\section{Acknowledgement}

The authors are very grateful to the anonymous referee how quickly she/he performed the reviewing task.

\section{References}

[1] G. Chartrand and L. Lesniak, Graphs \& Digraphs, second edition. Wadsworth \& Brooks/Cole Advanced Books and Software, Monterey, 1986.

[2] R. M. Figueroa-Centeno and R. Ichishima, The $n$-dimensional cube is felicitous, Bull. Inst. Combin. Appl., 41 (2004), 47-50.

[3] R. Figueroa-Centeno, R. Ichishima, and F. Muntaner-Batle, Magical coronations of graphs, Australas. J. Combin., 26 (2002), 199-208.

[4] R. M. Figueroa-Centeno, R. Ichishima, and F. A. Muntaner-Batle, Labeling the vertex amalgamation of graphs, Discuss. Math. Graph Theory, 23 (2003), 129-139.

[5] R. Figueroa-Centeno, R. Ichishima, F. Muntaner-Batle and A. Oshima, A magical approach to some labeling conjectures, Discuss. Math. Graph Theory, 31 (2011), 79-113. 
[6] J. A. Gallian, A dynamic survey of graph labeling, Electron. J. Combin. (2018), \#DS6.

[7] R. L. Graham and N. J. Sloane, On additive bases and harmonious graphs, SIAM J. Alg. Discrete Math., 1 (1980), 382-404.

[8] S. W. Golomb, How to number a graph, in Graph Theory and Computing, R. C. Read, ed., Academic Press, New York (1972), 23-37.

[9] R. Ichishima and A. Oshima, On partitional and other related graphs, Math. Comput. Sci., 5 (2011), 41-50.

[10] A. Kotzig, Decomposition of complete graphs into isomorphic cubes, J. Combin. Theory, Series B, 31 (1981), 292-296.

[11] S. M. Lee, E. Schmeichel, and S. C. Shee, On felicitous graphs, Discrete Math., 93 (1991), 201-209.

[12] K. Manickam, M. Marudai, and R. Kala, Some results on felicitous labeling of graphs, $J$. Combin. Math. Combin. Comput., 81 (2012), 273-279.

[13] A. Rosa, On certain valuations of the vertices of a graph, Theory of Graphs (Internat. Symposium, Rome, July 1966), Gordon and Breach, N. Y. and Dunod Paris (1967), 87-95.

[14] M. A. Seoud and E. A. Elsahawi, On almost graceful, felicitous and elegant graphs, J. Egyptian Math. Soc., 7 (1999), 137-149.

[15] V. Yegnanarayanan, On some additive analogues of graceful theme: cycle related graphs, Southeast Asian Bull. Math., 23 (1999), 1-17. 